\title{
Further evidence for preaxial hallucal polydactyly as a marker of diabetic embryopathy
}

\author{
Jennie Slee, Jack Goldblatt
}

\begin{abstract}
Maternal diabetes has an established aetiological link with developmental abnormalities, and the prevalence of major congenital malformations in the offspring of affected women is approximately $4-8 \%$, compared to the general population risk of about $3 \%$. Hallucal polydactyly, particularly with an unusual proximal placement of the extra digit, has been reported as a distinctive anomaly in diabetic embryopathy.

We report on a child of a diabetic mother with this unusual form of hallucal polydactyly, together with other skeletal anomalies, confirming that this malformation is a useful clinical marker for the diagnosis of diabetic embryopathy. (F Med Genet 1997;34:261-263)
\end{abstract}

Keywords: maternal diabetes; preaxial polydactyly; caudal regression.

Congenital malformations account for $50 \%$ of the perinatal mortality in infants of diabetic mothers, ${ }^{1-3}$ with the highest frequency in poorly controlled insulin dependent diabetes. Previous studies have documented a two to four fold increased risk of congenital malformations, but a recent epidemiological survey showed a four to seven fold increase in the incidence of skeletal, cleft lip/palate, neural tube, and cardiac abnormalities, being higher among female than male offspring. ${ }^{3}$ Confirming the role of maternal diabetes in the aetiology of the abnormalities can be difficult, given their lack of specificity. A report by Carey et al $l^{4}$ of a possible causal relationship between hallucal polydactyly, with a very unusual proximal placement of the extra digit, and diabetic embryopathy suggested a useful diagnostic marker of teratogenic effect in infants with multiple congenital abnormalities.

We report on a child with multiple skeletal abnormalities, including preaxial polydactyly, who was born to a woman with poorly controlled insulin dependent diabetes. The phenotype is described and depicted and the unusual nature of the hallucal polydactyly is discussed, to provide further evidence that this anomaly is an important clinical marker of diabetic embryopathy.

\section{Case report}

A 2 year old female was the third child of a non-consanguineous young couple. The mother had been an insulin dependent diabetic from the age of 12 years and the pregnancy, particularly the periconceptional period, was complicated by poor control of her diabetes, and her blood $\mathrm{HbA} 1 \mathrm{c}$ was $12.1 \%$ when she was initially seen at 11 weeks. A non-elective caesarean section was performed at 37 weeks' gestation in view of decreased fetal movements and an abnormal cardiotocograph. Apgar scores were 7 and 9 at one and five minutes respectively, but she developed early onset hyaline membrane disease requiring ventilation for two days. She was macrosomic, with a birth weight of $4090 \mathrm{~g}$, and was noted to have multiple skeletal abnormalities. These included preaxial polydactyly of the right foot with an unusual proximally placed extra hallux (figs 1 and 2), a shortened dysplastic right lower leg, camptodactyly of the right middle finger, and scoliosis convex to the right. The remainder of the clinical examination was unremarkable.

Both hips were dislocated, with no evidence of acetabular dysplasia, and radiographic examination of the right leg (fig 3 ) showed a normal femur and tibial hypoplasia with consequent derangement of the knee and ankle, with the fibular head being dislocated superiorly. The talus on that side was small, and the calcaneum rounder than normal. There was polydactyly with the duplicated hallux apparently articulating with the medial side of the first metatarsal and posteriorly directed

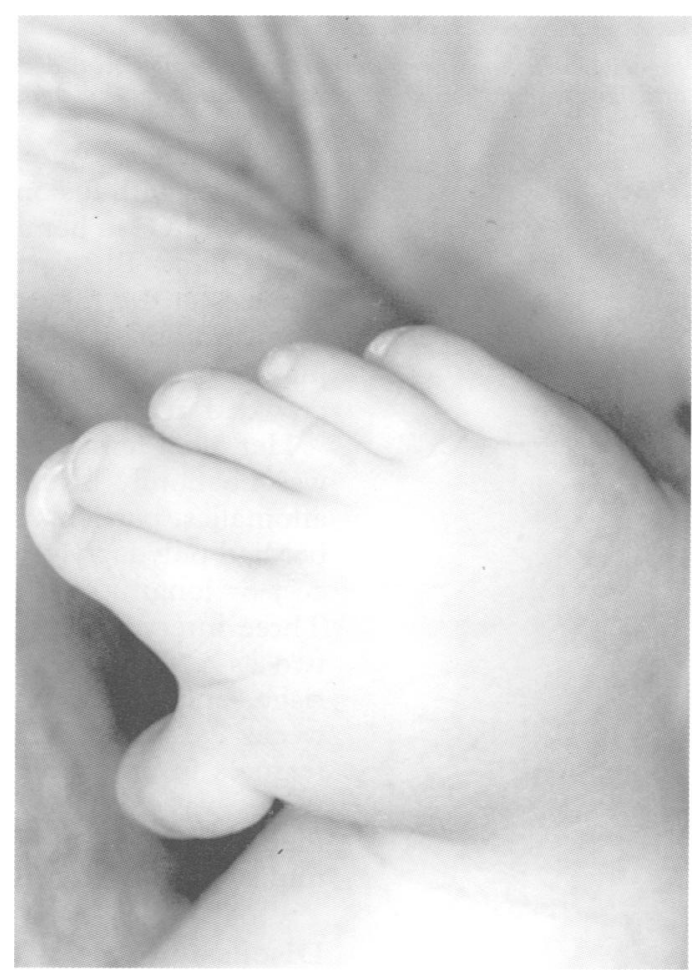

Figure 1 Dorsal aspect of the right foot illustrating the proximal placement of the duplicated hallux.
Received 17 September 1996 Revised version accepted for 1996 


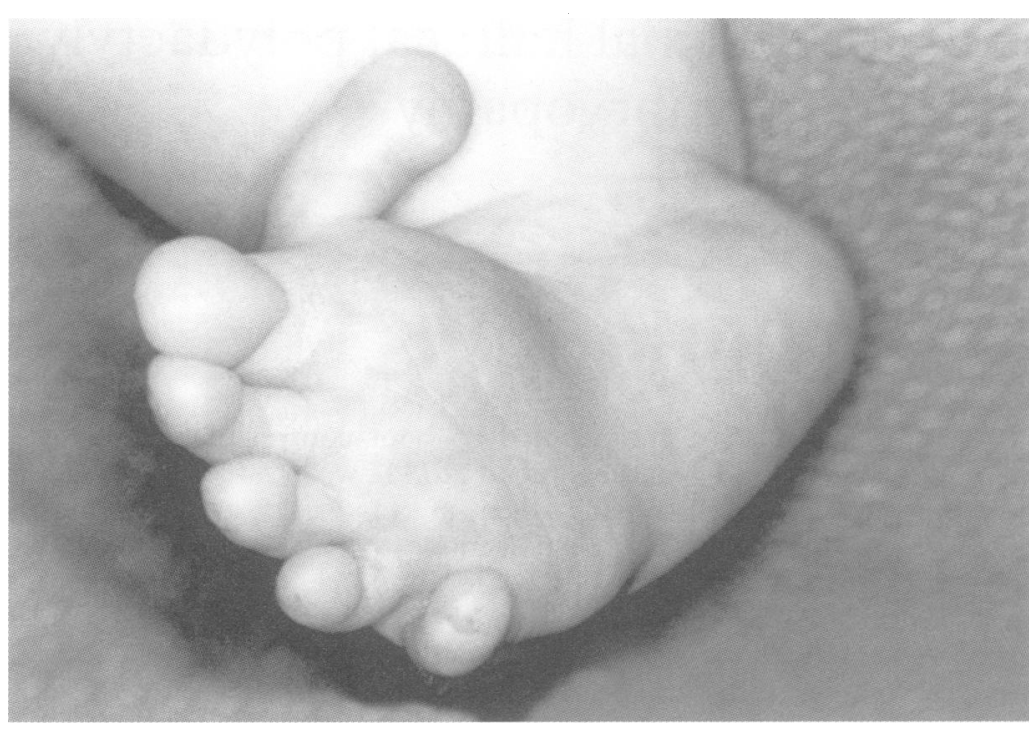

Figure 2 Plantar aspect of the right foot showing the posterior direction of the proximally placed hallucal polydactyly.

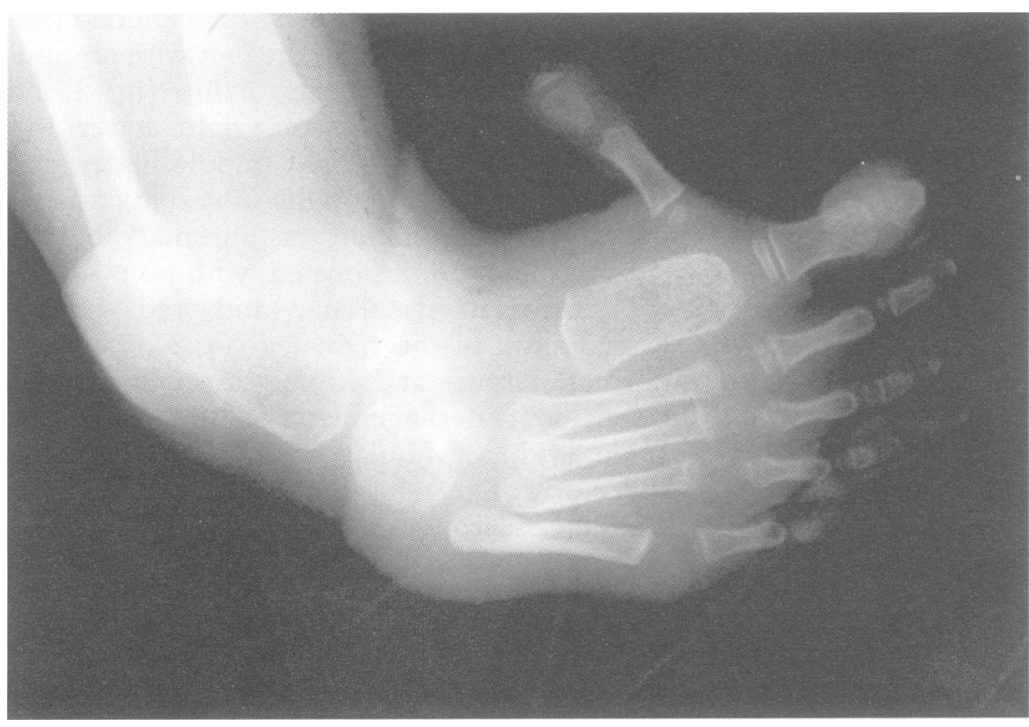

Figure 4 Anteroposterior radiograph of the right foot. The duplicated hallux appears to articulate with the medial aspect of the first metatarsal and is posteriorly directed.

(fig 4). There were multiple vertebrocostal anomalies with fusion of the spinous processes of $\mathrm{C} 2$ and $\mathrm{C} 3$, a hemivertebra at $\mathrm{T} 11$, dysplastic $\mathrm{T} 10$ and $\mathrm{T} 12$ vertebrae, lordosis at $\mathrm{T} 9-11$, scoliosis at the thoracolumbar junction, and a hemivertebra at S5 with absent left lateral masses of S3-5. The ribs were dysplastic and crowded in an irregular manner.

Her older female sibs were normal and there was no family history of any similar skeletal anomalies, particularly no other family member had polydactyly, or broad thumbs or halluces, or longitudinal split defects in the nails. Three intervening pregnancies had been lost, two as a result of early spontaneous miscarriage, and one a fetal death in utero at 15 weeks' gestation. There were no dysmorphic features in this latter fetus. In all pregnancies there was similarly poor periconceptional metabolic control of the diabetes, which improved only after the first trimester.

\section{Discussion}

Carey $e t a l^{4}$ evaluated six subjects with preaxial polydactyly of the feet born to diabetic

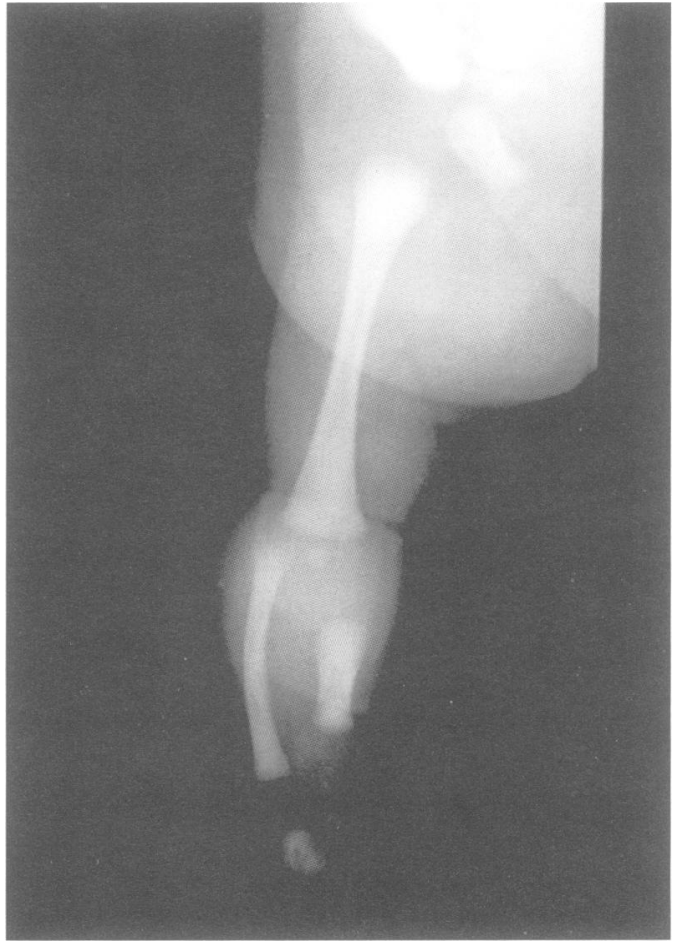

Figure 3 Anteroposterior radiograph of the right leg. The hip is dislocated, but the acetabulum is not dysplastic. The tibia is hypoplastic and the fibular head is superiorly displaced, with derangement of the knee and ankle. The talus is small and the calcaneum rounded.

mothers, and five showed an unusual proximal placement of the extra hallux. Martínez-Frías et at confirmed the association of hallucal polydactyly in infants of diabetic mothers, but not of the distinctive proximal variant described by Carey et al. ${ }^{4}$ These cases also serve to illustrate the phenomenon that teratogen induced birth defects are not always reduction defects but can also result in duplication, the mechanism of which has yet to be determined.

In view of the background prevalence of birth defects, it is often difficult to confirm the relationship between a possible teratogen and a congenital malformation. The birth defects associated with maternal diabetes are multiple and non-specific, apart from the caudal regression syndromes, of which up to $50 \%$ are reported to occur in infants of diabetic mothers. ${ }^{67}$ Counselling in individual cases is thus complicated in terms of both recurrence risks and the emotional parental burden of implicating the maternal condition in the aetiology.

This female child is the sixth reported case documenting an unusual proximal placement of an extra hallux in a child born to an insulin dependent diabetic mother, whose diabetes was poorly controlled during pregnancy. This finding supports the hypothesis of Carey et $a l^{4}$ that this form of hallucal polydactyly is a useful, albeit rare, marker of the teratogenic effect of maternal diabetes, and a flag to search for other potential associated birth defects. Further structured reporting on this relationship, in particular case controlled studies, may help clarify this issue. 
1 Reece A, Hobbins J. Diabetic embryopathy: pathogenesis, prenatal diagnosis and prevention. Obstet Gynecol Surv 1986;41:325-35

2 Becerra J, Khoury M, Cordero J, et al. Diabetes mellitus during pregnancy and the risks for specific birth defects: a population based case-control study. Pediatrics 1990;85:1-9.

3 Janssen PA, Rothman I, Schwartz SM. Congenital malformations in newborns of women with established and gestational diabetes in Washington State, 1984-91. Pediatr Perinat Epidemiol 1996;10:52-63.

4 Carey JD, Hommell M, Fineman RM, Hall BD. Hallucal polydactyly in infants of diabetic mothers: a clinical marker and possible clue to teratogenesis. Proc Greenwood Genet Cent 1990;9:95.

5 Martinez-Frias ML, Bermejo E, Cereijo A. Preaxial polydactyly of feet in infants of diabetic mothers: epidemiological test of a clinical hypothesis. $\mathrm{Am} \mathrm{f} \mathrm{Med}$ Genet 1992;42:643-6.

6 Duncan PA, Shapiro LR, Klein RM. Sacrococcygeal dysgenesis association. $A m$ f Med Genet 1991;41:153-61.

7 Phillips WA, Cooperman DR, Lindquest TC, Sullivan RC, Miller EA. Orthopaedic management of lumbosacral agenesis. F Bone foint Surg Am 1982;64:1282-94. 